Article

\title{
Shannon (Information) Measures of Symmetry for 1D and 2D Shapes and Patterns
}

\author{
Edward Bormashenko ${ }^{1 *}$, Irina Legchenkova ${ }^{1}$, Mark Frenkel ${ }^{1}$, Nir Shvalb $^{2}$, Shraga Shoval ${ }^{3}$ \\ ${ }^{1}$ Chemical Engineering Department, Engineering Faculty, Ariel University, P.O.B. 3, 407000 Ariel, Israel \\ ${ }^{2}$ Department of Mechanical Engineering \& Mechatronics, Faculty of Engineering, Ariel University, P.O.B. \\ 3, Ariel 407000, Israel \\ ${ }^{3}$ Department of Industrial Engineering and Management, Faculty of Engineering, Ariel University, \\ P.O.B. 3, Ariel 407000, Israel \\ * Correspondence: edward@ariel.ac.il
}

Abstract: Informational (Shannon) measures of symmetry are introduced and analyzed for the patterns built of 1D and 2D shapes. The informational measure of symmetry $\boldsymbol{H}_{\text {sym }}(\boldsymbol{G})$ characterizes the an averaged uncertainty in the presence of symmetry elements from the group $G$ in a given pattern; whereas the Shannon-like measure of symmetry $\boldsymbol{\Omega}_{\text {sym }}(\boldsymbol{G})$ quantifies averaged uncertainty of appearance of shapes possessing in total $n$ elements of symmetry belonging to group $G$ in a given pattern. $\boldsymbol{H}_{\text {sym }}\left(\boldsymbol{G}_{\mathbf{1}}\right)=\boldsymbol{\Omega}_{\text {sym }}\left(\boldsymbol{G}_{\mathbf{1}}\right)=\mathbf{0}$ for the patterns built of irregular, non-symmetric shapes. Both of informational measures of symmetry are intensive parameters of the pattern and do not depend on the number of shapes, their size and area of the pattern. They are also insensitive to the long-range order inherent for the pattern. Informational measures of symmetry of fractal patterns are addressed. The mixed patterns including curves and shapes are considered. Time evolution of

Citation: Lastname, F.; Lastname, F.; Lastname, F. Title. Appl. Sci. 2021, 11, x. https://doi.org/10.3390/xxxxx

Academic Editor: Firstname Lastname

\section{Received: date}

Accepted: date

Published: date

Publisher's Note: MDPI stays neutral with regard to jurisdictional claims in published maps and institutional affiliations.

Copyright: (c) 2021 by the authors. Submitted for possible open access publication under the terms and conditions of the Creative Commons Attribution (CC BY) license (https://creativecommons.org/license s/by/4.0/). the Shannon measures of symmetry is treated. The close-packed and dispersed 2D patterns are analyzed.

Keywords: informational measure of symmetry; 1D shapes; 2D shapes; fractal patterns; time evolution; symmetry; pattern.

\section{Introduction}

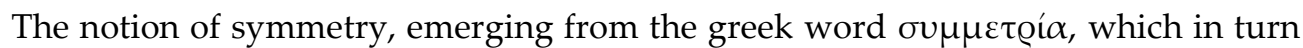
means agreement in dimensions, arrangement, plays a crucial and instructive role in mathematics and natural sciences [1-5], generating the conservation laws [6] and being crtitically important in materials science [7], physics [2, 5, 6], quantum theory [8], quantum chemistry and spectroscopy [9] and, of course, crystallography [10-11]. Ideas, methods and techniques arising from spatial symmetry are of a basic importance in the philosophy and psychology of aesthetics [12-15].

Our paper is devoted to the quantification of symmetry. Symmetry is conventionally described in a contrariety, binary manner, implying that the system is either completely symmetric or completely asymmetric. Fang et al. involved the group theoretical approach to overcome this dichotomous problem, and introduced the degree of symmetry as a nonnegative continuous number ranging from zero to unity [16]. The deviation from the per-
14 15 16 17 18 19 20 21 22 23 24 
fect geometric symmetry was quantified with the "continuous measure of symmetry" introduced by Avnir, Zabrodsky and co-workers in refs. 17-21. The continuous measure of symmetry was defined as the minimal average square displacement of the points, that the shape has to undergo in order to attain the prescribed symmetry [17-21].

On the other hand, ordering in 2D patterns is usually quantified with Voronoi entropy, is given by:

$$
S_{\text {vor }}=-\sum_{i} P_{i} \ln P_{i},
$$

where $P_{i}$ is the portion of the polygons possessing $n$ edges in a given Voronoi diagram (also called the coordination number of the polygon) and $i$ is the total number of polygon types with different number of edges (see refs. 22-24). In our recent papers we demonstrated that the continuous measure of symmetry introduced in refs, 20-21 and the Voronoi entropy of the given 2D pattern are not necessarily correlated [25-26]. Our paper is devoted to the alternative quantification of symmetry with the Shannon-like informational measure of symmetry which was first introduced in ref. 27, in which it was applied to the quantitative analysis of Voronoi diagrams arising from the Penrose tiling. In our present paper we generalize and develop the notion of the informational measure of symmetry. An interpretation of the notion of the informational measure of symmetry follows the approach suggested in ref. 28, namely, it is understood as an averaged uncertainty in the presence of symmetry elements from the group $G$ in the given pattern [27-28]. We demonstrate that different approaches to the calculation of the informational measure of symmetry are possible.

\section{Results and discussion}

Consider 2D pattern build of 1D and/or 2D shapes or lines, demonstrating a number of symmetry elements (rotational symmetry; centers of symmetry, axes of symmetry, etc.), denoted $\boldsymbol{G}_{\boldsymbol{i}}, \boldsymbol{i}=\mathbf{1}, \mathbf{2} \ldots \boldsymbol{k}$, where $k$ is a number of nonidentical symmetry operations. Elements $\boldsymbol{G}_{\boldsymbol{i}}$ form the symmetry group of the shape $G$ (which should be clearly distinguished from the symmetry group of the entire pattern). Thus, the informational measure of symmetry of the pattern (abbreviated IMS) will be defined in a Shannon-like form as:

$$
H_{\text {sym }}(G)=-\sum_{i=1}^{k} P_{i}\left(G_{i}\right) \ln P_{i}\left(G_{i}\right),
$$

where $P_{i}\left(G_{i}\right)$ is the probability of appearance of the symmetry operation $G_{i}$ within the shapes (lines) constituting the pattern, defined as:

$$
P_{i}\left(G_{i}\right)=\frac{m\left(G_{i}\right)}{N_{G}} \leq 1,
$$

where $N_{G}=\sum_{i=1}^{k} m\left(G_{i}\right)$ is a total number of symmetry elements (operations) appearing in the 1D or 2D shapes recognized in a given pattern and $m\left(G_{i}\right)$ is a number of the same symmetry elements (operations) $G_{i}$ calculated for a given pattern. The normalization condition given by Eq. 4 takes place:

$$
\sum_{i=1}^{k} P_{i}\left(G_{i}\right)=1
$$

Alternatively, the symmetry of the pattern may be quantified with the parameter $\boldsymbol{\Omega}_{\boldsymbol{s y m}}(\boldsymbol{G})$, defined according to Eq. 5:

$$
\left.\mathbf{\Omega}_{s y m}(G)=-\sum_{n=1}^{l} P_{n}(n(G))\right) \ln P_{n}(n(G))
$$

where $n$ denotes the total number of elements of symmetry recognized in a shape; $n$ changes from unity to the maximal number of elements of symmetry inherent for the shapes appearing in the pattern and denoted "l", $\boldsymbol{P}_{\boldsymbol{n}}(\boldsymbol{n}(\boldsymbol{G}))$ is the probability to find within the pattern the shape possessing in total $n$ elements of symmetry belonging to group $G$, defined as:

$$
P_{n}(n(G))=\frac{r(n(G))}{N_{S}}
$$

61 62 63 64 
where $r\left(n(G)\right.$ ) is a number of shapes possessing in total $n$ elements of symmetry, and $N_{S}$ is the total number of shapes in a given pattern. The normalization condition given by Eq. 7 takes place:

$$
\sum_{n=1}^{l} P_{n}(n(G))=1
$$

The Shannon-shaped measure $\boldsymbol{\Omega}_{\text {sym }}(\boldsymbol{G})$ is interpreted, in turn, as an averaged across the pattern uncertainty to find within a given pattern the shape possessing in total $n$ elements of symmetry [27-28]. The definition of the Shannon measure of symmetry $\boldsymbol{\Omega}_{\text {sym }}(\boldsymbol{G})$ supplied by Eqs. 5-7 resembles the definition of the Voronoi entropy, supplied by Eq. 1 with the following difference: instead of number of the polygon edges, appearing in the definition of the Voronoi entropy, we exploit in our definition the number of elements of symmetry $n$ in the shape; thus, we label this measure as the Voronoi-Shannon measure of symmetry and abbreviate VSMS. The definition of VSMS supplied by Eqs. 5-7 latently implies that more symmetrical shape is characterized by the larger number of symmetry elements. We will illustrate the difference between the informational measure of symmetry (IMS) and the Voronoi-Shannon measure of symmetry (VSMS) with the numerous examples.

\section{2. Informational measures of symmetry of the patterns built of $1 \mathrm{D}$ objects}

Consider first the informational measure of symmetry (IMS) and the Voronoi-Shannon measure of symmetry (VSMS) of the patterns comprising only 1D objects (lines). Let us start from the pattern built of $p$ irregular lines, such as depicted in Figure 1. The analysis should necessarily start from the establishment of the symmetry group of the pattern. In this case we recognize for all of the shapes constituting the pattern the single element of symmetry, namely the 1fold rotational symmetry reduced to the rotation by the angle $\varphi_{1}=k \frac{2 \pi}{1}=2 \pi$, denoted $G_{1}$.

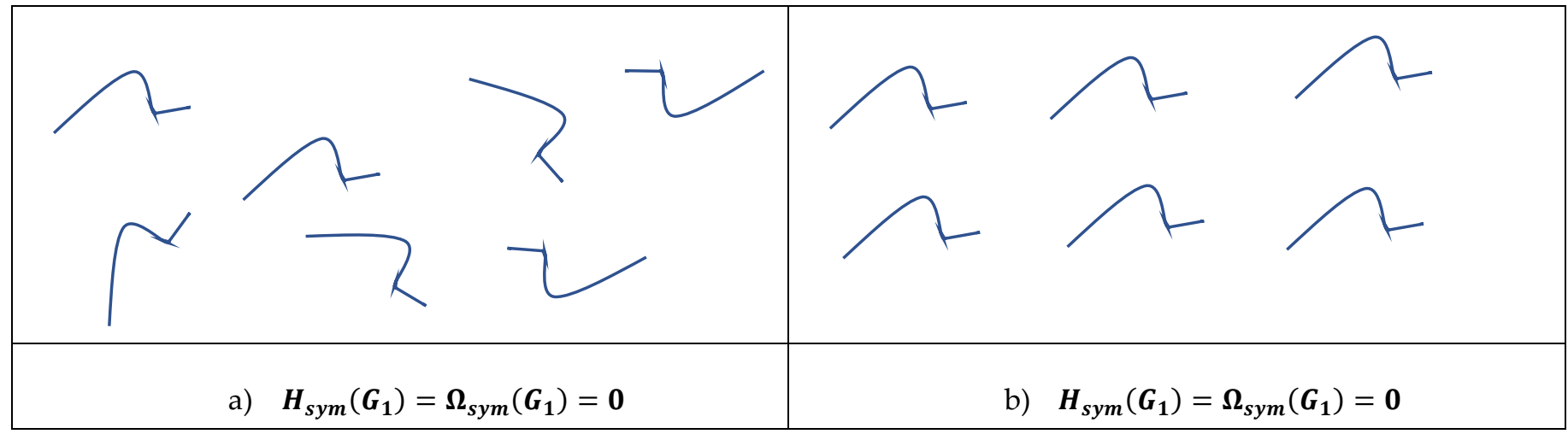

Figure 1. Pattern built of the 1D irregular (non-symmetrical) lines. a) Random pattern; $\mathbf{b}$ ) regular pattern demonstrating the translational symmetry.

Let us calculate first $\boldsymbol{H}_{\text {sym }}$ for the pattern, shown in Figure 1a. In this case $\boldsymbol{N}_{\boldsymbol{G}}=\boldsymbol{p}$; thus, $\boldsymbol{P}\left(\boldsymbol{G}_{\mathbf{1}}\right)=\mathbf{1}$ and consequently

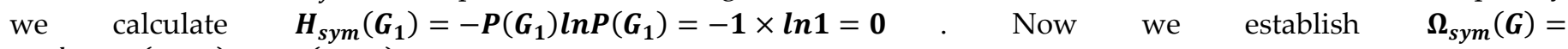
$\left.-\sum_{n=1}^{l} \boldsymbol{P}_{\boldsymbol{n}}(\boldsymbol{n}(\boldsymbol{G}))\right) \boldsymbol{l n} \boldsymbol{P}_{\boldsymbol{n}}(\boldsymbol{n}(\boldsymbol{G}))$. It is easily seen that for the irregular shapes possessing a single element of symmetry $\boldsymbol{P}_{\boldsymbol{n}}(\boldsymbol{n}=\mathbf{1})=\mathbf{1}$, hence $\boldsymbol{\Omega}_{\text {sym }}\left(\boldsymbol{G}_{1}\right)=\mathbf{0}$. The same conclusion, i.e. $\boldsymbol{H}_{\text {sym }}\left(\boldsymbol{G}_{\mathbf{1}}\right)=\boldsymbol{\Omega}_{\text {sym }}\left(\boldsymbol{G}_{\mathbf{1}}\right)=\mathbf{0}$ holds from the pattern built from non-identical, irregular, non-symmetrical lines. And it should be emphasized that the same conclusion. i.e. $\boldsymbol{H}_{\text {sym }}\left(\boldsymbol{G}_{\mathbf{1}}\right)=\boldsymbol{\Omega}_{\text {sym }}\left(\boldsymbol{G}_{\mathbf{1}}\right)=\mathbf{0}$ is true for the regular pattern, comprising irregular, non-symmetrical lines, such as shown in Figure 1b, demonstrating the pattern characterized by the translational symmetry. Thus, we concluded that both of introduced measures of symmetry are insensitive to the long-range order. How the obtained result should be interpreted? $\boldsymbol{H}_{\text {sym }}\left(\boldsymbol{G}_{\mathbf{1}}\right)=\mathbf{0}$ means that an averaged uncertainty to reveal symmetry operation $\boldsymbol{G}_{\mathbf{1}}$ within the pattern is zero; indeed, all of the 1D shapes depicted in Figure 1 posses this element of symmetry; in turn, $\boldsymbol{\Omega}_{\text {sym }}\left(\boldsymbol{G}_{\mathbf{1}}\right)=\mathbf{0}$ means that an averaged uncertainty to find within the pattern the shape demonstrating the single element of symmetry is zero; indeed, every shape in the pattern has in total only one element of symmetry, namely $\boldsymbol{G}_{\mathbf{1}}$ (see ref. 28).

Now consider the patterns composed of the straight line segments, shown in Figures 2a-b. The symmetry group of the straight line segments is built of two elements, namely the unity element which is the 1-fold rotational symmetry, 
which is the rotation by the angle $\varphi_{1}=\boldsymbol{k} \frac{2 \pi}{1}=\mathbf{2 \pi}$ (denoted $G_{1}$ ) and the 2-fold rotational symmetry the angle $\varphi_{1}=$ $\boldsymbol{k} \frac{2 \pi}{2}=\boldsymbol{\pi}$ (which may be changed by the mirror axis), denoted $\boldsymbol{G}_{2}$. Let us calculate $\boldsymbol{H}_{\text {sym }}$. For the pattern, built of the $p$ segments we obtain $P\left(G_{1}\right)=P\left(G_{2}\right)=\frac{p}{2 p}=\frac{1}{2^{\prime}}$ thus, $H_{\text {sym }}=-2 \frac{1}{2} \ln \frac{1}{2}=0.69$.

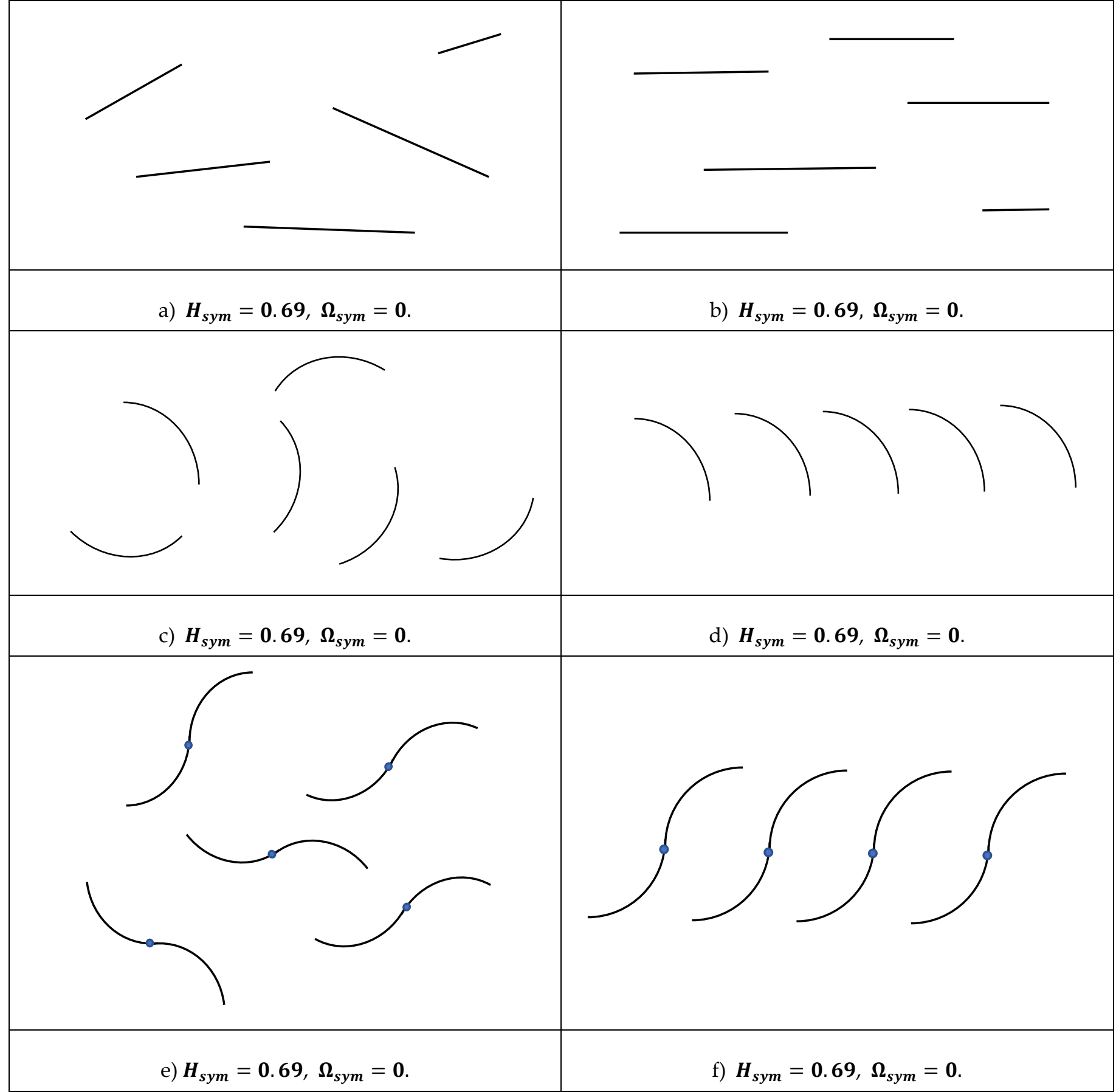

Figure 2. Patterns built of the shapes possessing two elements of symmetry. a) random straight line segments, b) ordered straight line segments, c) random arcs of a circle; d) ordered arcs of a circle, e) random symmetrical segments of a cubic parabola, f) ordered symmetrical segments of a cubic parabola. Center of symmetry is shown with a blue circle.

Now, let us establish $\left.\boldsymbol{\Omega}_{\text {sym }}(\boldsymbol{G})=-\sum_{\boldsymbol{n}=\mathbf{1}}^{\boldsymbol{l}} \boldsymbol{P}_{\boldsymbol{n}}(\boldsymbol{n}(\boldsymbol{G}))\right) \boldsymbol{I n} \boldsymbol{P}_{\boldsymbol{n}}(\boldsymbol{n}(\boldsymbol{G}))$. For the patterns depicted in Figures 2a-b we have the shapes, which one of them has two aforementioned elements of symmetry; thus, $\boldsymbol{P}_{\mathbf{2}}(\boldsymbol{n}=\mathbf{2})=\mathbf{1}$, hence $\boldsymbol{\Omega}_{\boldsymbol{s y m}}=\mathbf{0}$. 
And, again, obviously both of the introduced measures of symmetry coincide for the patterns presented in Figures 2a$\mathbf{b}$, and they are insensitive to the long-range order of segments, shown in Figure $\mathbf{2} \mathbf{b}$.

Consider the patterns built of the $p$ arcs of a circle (or, perhaps, the symmetric segments of the parabola $\boldsymbol{y}=$ $\boldsymbol{\alpha} \boldsymbol{x}^{2}, \boldsymbol{\alpha}=$ const), shown in Figures $2 \mathrm{c}-\mathrm{d}$. The symmetry group of the shapes depicted in Figures $2 \mathrm{c}-\mathrm{d}$ is built of two elements, namely the unity element which is the 1-fold rotational symmetry (denoted $\boldsymbol{G}_{\mathbf{1}}$ ) and the mirror axis, denoted $\boldsymbol{G}_{2}$. For this kind of pattern we obtain $\left(\boldsymbol{G}_{1}\right)=\boldsymbol{P}\left(\boldsymbol{G}_{2}\right)=\frac{p}{2 \boldsymbol{p}}=\frac{\mathbf{1}}{\mathbf{2}}$, thus, $\boldsymbol{H}_{\boldsymbol{s y m}}=-\mathbf{2} \frac{\mathbf{1}}{\mathbf{2}} \boldsymbol{\boldsymbol { n }} \frac{\mathbf{1}}{\mathbf{2}}=\mathbf{0 . 6 9}$. Consequently, in this case $\boldsymbol{\Omega}_{\text {sym }}=\mathbf{0}$ (due to the $\boldsymbol{P}_{\mathbf{2}}(\boldsymbol{n}=\mathbf{2})=\mathbf{1}$, and $\boldsymbol{I n} \boldsymbol{P}_{\mathbf{2}}=\mathbf{0}$ ); the long range order, shown in Figure 2d plays no role in the calculation of both of measures of symmetry. Now consider the patterns built of the $p$ symmetric segments of cubic parabola $\boldsymbol{y}=\boldsymbol{\alpha} \boldsymbol{x}^{\mathbf{3}}, \boldsymbol{\alpha}=\mathbf{c o n s t}$ ) shown in Figures 2e-f. The considerations akin to the aforementioned ones immediately yield: $\boldsymbol{H}_{\text {sym }}=\mathbf{0 . 6 9}, \boldsymbol{\Omega}_{\text {sym }}=\mathbf{0}$. Thus, we come to the conclusion: the all of the patterns depicted in Figure 2 are equivalent from the point of view of the introduced Shannon measures of symmetry, namely, $\boldsymbol{H}_{\boldsymbol{s} y \boldsymbol{m}}$ and $\boldsymbol{\Omega}_{\boldsymbol{s y m}}$. Moreover, VSMS of the patterns depicted in Figure $\mathbf{1}$ and Figure $\mathbf{2}$ is equal, however IMS of the patterns shown in Figure $\mathbf{1}$ and Figure $\mathbf{2}$ is different. It should be emphasized, that both of the Shannon and the Voronoi-Shannon informational measures of symmetry of the discussed patterns are the intensive properties of the patterns and they are independent of the area of the pattern or the density and size of the shapes, and in this sense they are different from the true thermodynamic entropy, which is an extensive parameter of the system. And it should be emphasized that the long-range order does not influence both of the informational measures of symmetry.

Now consider the mixed patterns, comprising 1D objects (lines), depicted in Figure 3.

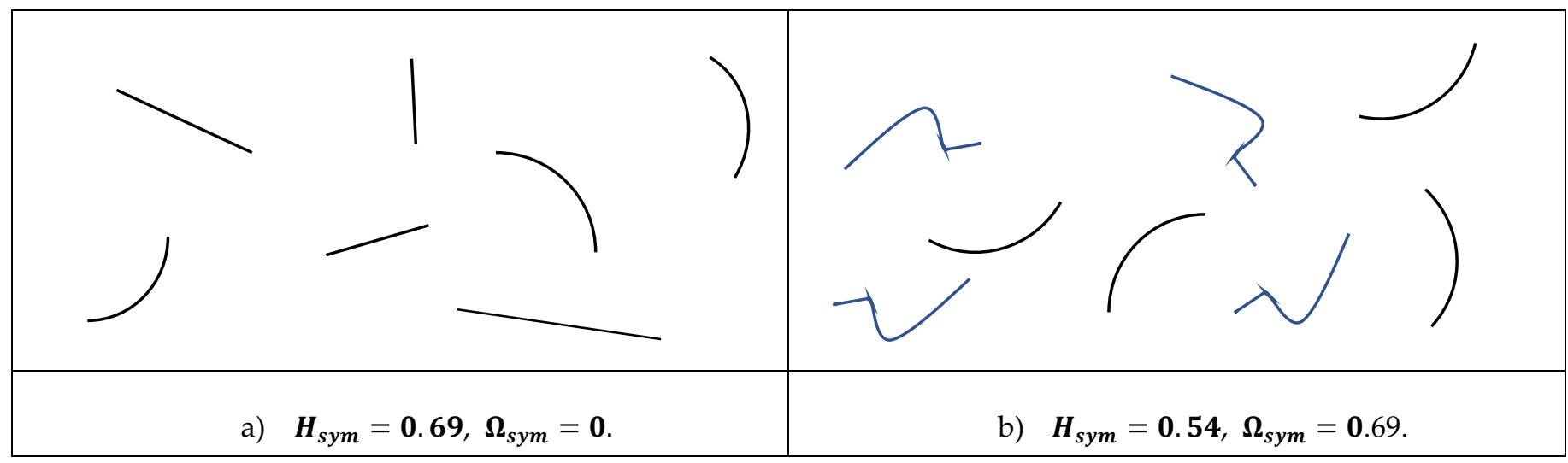

Figure 3. The mixed patterns comprising 1D objects are depicted. a) the pattern is built from the straight line segments and the arcs of a circle, $b$ ) the pattern is composed of the irregular (non-symmetrical) shapes and arcs of a circle.

Let us start from the pattern comprising the straight line segments and the arcs of a circle, shown in Figure 3a. Both of the shapes are characterized by the same symmetry group; namely, the symmetry group comprises the unity element which is the 1-fold rotational symmetry, and the 2-fold rotational symmetry (which may be changed by the mirror axis), thus, $\boldsymbol{H}_{\text {sym }}=-\mathbf{2} \frac{\mathbf{1}}{\mathbf{2}} \boldsymbol{\operatorname { l n }} \frac{\mathbf{1}}{\mathbf{2}}=\mathbf{0 . 6 9}$. Both of the shapes have in total two elements of symmetry, hence $\boldsymbol{\Omega}_{\text {sym }}=$ 0. Now consider the pattern built from $p$ irregular, non-symmetrical curves and $p$ arcs of a circle, presented in Figure 3b. We recognize two elements of symmetry in this pattern, namely the 1 fold rotational symmetry inherent for all of the shapes and denoted $\boldsymbol{G}_{\mathbf{1}}$ and the mirror axis labeled $\boldsymbol{G}_{\mathbf{2}}$ inherent for the arcs only. Thus, the entire number of the symmetry elements in the pattern $\boldsymbol{N}_{G}=\mathbf{3 p}$ (see Eq. 3); consequently we easily calculate $\boldsymbol{P}\left(\boldsymbol{G}_{1}\right)=\frac{2 p}{3 p}=\frac{2}{3} ; \boldsymbol{P}\left(\boldsymbol{G}_{2}\right)=\frac{p}{3 p}=$ $\frac{1}{3}$ and finally we obtain $\boldsymbol{H}_{\text {sym }}=-\left(\frac{2}{3} \ln \frac{2}{3}+\frac{1}{3} \ln \frac{1}{3}\right)=\mathbf{0 . 5 4}$. Now let us calculate $\boldsymbol{\Omega}_{\text {sym }}$ for the mixed pattern, shown in Figure $3 b$. For this pattern we calculate $\left(N_{s}=2 p\right): P_{1}(n=1)=\frac{p}{2 p}=\frac{1}{2} ; P_{2}(n=2)=\frac{p}{2 p}=\frac{1}{2} ;$ thus, $\Omega_{s y m}=0.69$.

\section{2. Informational measures of symmetry of the patterns built of $2 \mathrm{D}$ shapes}


Now we address the patterns composed of the 2D shapes. Let us start from the completely disordered pattern, built of $p$ irregular shapes depicted in Figure 4a. In this case we recognize for all of the non-symmetrical shapes constituting the pattern the single element of symmetry, namely the rotation $\varphi_{1}=k \frac{2 \pi}{1}=2 \pi ;$ thus, $N_{G}=p ;$ thus, $P\left(G_{1}\right)=1$ and consequently $H_{\text {sym }}=-\sum_{i=1}^{1} P\left(G_{i}\right) \ln P\left(G_{i}\right)=\sum_{i=1}^{6} 1 \ln (1)=0$. It is easily seen that $\Omega_{\text {sym }}(G)=0$ takes place for the same pattern. Let us analyze the $2 \mathrm{D}$ pattern comprising $p$ identical equilateral triangles, depicted in Figure $4 \mathbf{b}$. The symmetry group of the equilateral triangle is the dihedral symmetry group labeled usually $D_{3}$. In the case of equilateral triangles, shown in Figure $4 \mathrm{~b}$ we have $N_{g}=6 p$ elements of symmetry, which are $3 p$ symmetry axes and $3 p$ rotations. IFM calculated with Eq. 2 equals $H_{\text {sym }}\left(D_{3}\right)=1.792$. On the other hand, all of the shapes constituting the pattern shown in Figure $4 \mathbf{b}$ have the same number of the symmetry elements; thus, $\Omega_{\text {sym }}\left(D_{3}\right)=0$.

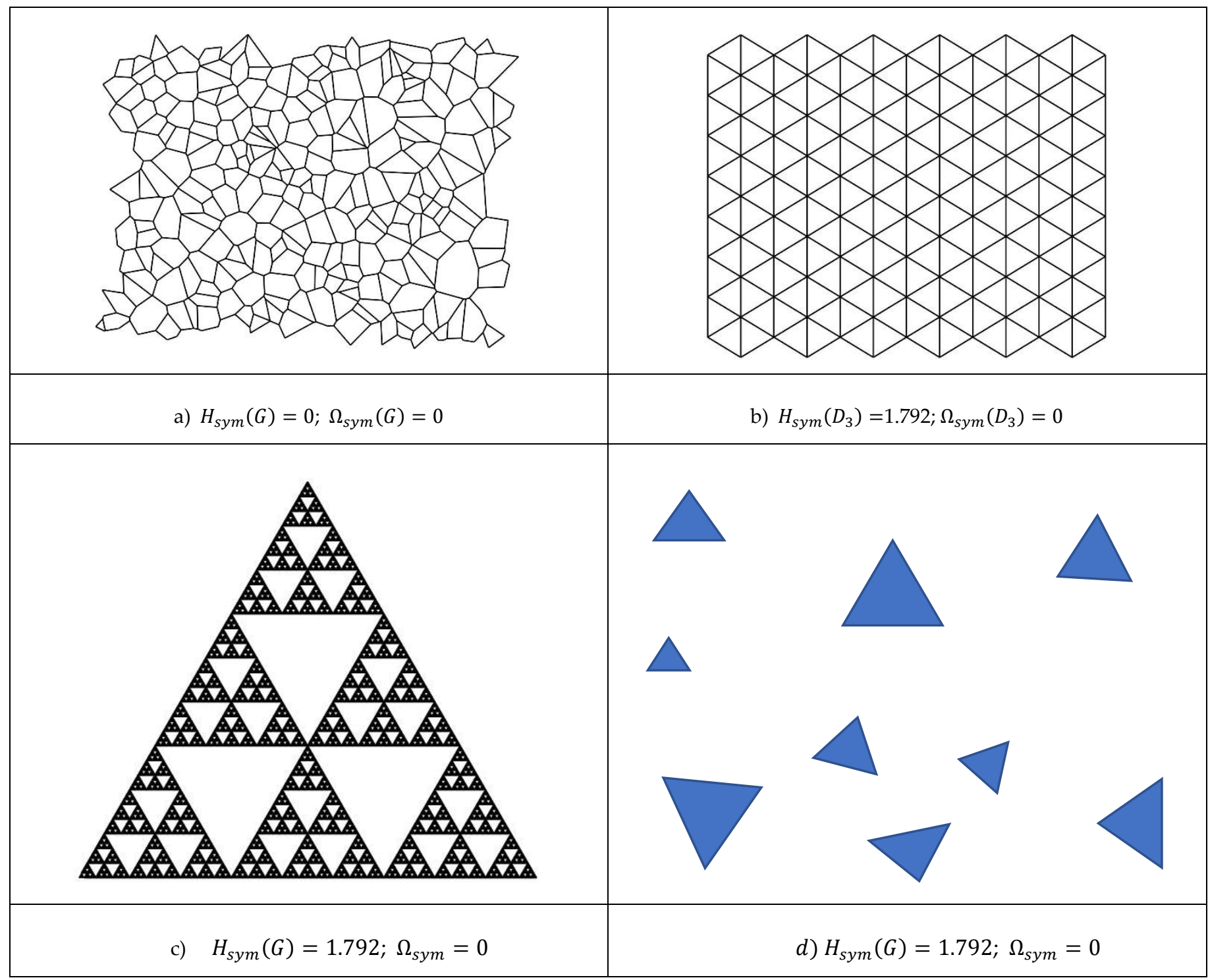

Figure 4. (a) Pattern filled with different unsymmetrical polygons, demonstrating: $H_{\text {sym }}(G)=0 ; \Omega_{\text {sym }}(G)=0$ is depicted. b) 2D pattern comprising identical equilateral triangles, demonstrating: $\left.H_{\text {sym }}(G)=1.792 ; \Omega_{\text {sym }}=0 . \mathrm{c}\right)$ Serpinski carpet built of equilateral triangles; d) pattern composed of the randomly dispersed equilateral triangles of 
Consider the fractal Serpinski carpet built of equilateral triangles, shown in Figure 4c [29-30]. It is easily seen, that $H_{\text {sym }}(G)=1.792 ; \Omega_{\text {sym }}=0$ for any scaling level of its fractal structure. Thus, we conclude, that an informational measures of symmetry are invariant with respect to the scaling of the Serpinski carpet. Obviously, the conclusion holds for the Serpinski carpet built of squares. The general problem of calculation of the informational measures of symmetry of fractal structures deserves an additional research. The pattern characterized by the informational measures of symmetry should not be necessarily close-packed, as shown in Figure $\mathbf{4 d}$, representing the set of randomly dispersed equilateral triangles of various areas. It is easily seen, that in this case $H_{\text {sym }}\left(D_{3}\right)=1.792, \Omega\left(D_{3}\right)=0$ takes place. Again, the introduced informational measures of symmetry are insensitive to the presence/absence of the long-range order in a given pattern.

It is noteworthy that the patterns should not be necessarily built of polygons; it may comprise curvilinear shapes such as ellipses, shown in Figure 5.

\begin{tabular}{|l|}
\hline a) $H_{\text {sym }}=1.1 ; \Omega_{\text {sym }}=0$ \\
\hline c) $H_{\text {sym }}=1.1 ; \Omega_{\text {sym }}=0$
\end{tabular}

Figure 5. Patterns built from the ellipses are shown. a) pattern built of the dispersed random ellipses of various sizes; b) pattern built of dispersed ordered identical ellipses; c) pattern comprising close-packed identical ellipses. Area between forms curvilinear quadrangle; d) pattern comprising close-packed identical ellipses. Areas between form curvilinear triangles.

Let us start from the pattern depicted in Figure 5a comprising the dispersed random ellipses of various sizes. The group of symmetry of ellipse includes 3 elements, namely: namely the 1 fold rotational symmetry denoted $G_{1}$ and two distinguishable mirror axes denoted correspondingly $G_{2}$ and $G_{3}$; obviously $P\left(G_{1}\right)=P\left(G_{2}\right)=P\left(G_{3}\right)=\frac{1}{3}$; thus, we calculate $H_{\text {sym }}=-3 \frac{1}{3} \ln \frac{1}{3}=1.1$. All of the ellipses have in total three axes of symmetry, hence $\Omega_{\text {sym }}=0$. The same is true for the dispersed identical long-range ordered ellipses, shown in Figure $5 \mathbf{b}$. The same conclusion holds for the close-packed ordered identical ellipses forming the patterns shown in Figure $5 \mathbf{c}-\mathbf{d}$. Figures $\mathbf{5}$ c-d illustrate the idea that the set of the shapes should be clearly defined. Indeed, $H_{\text {sym }}=1.1 ; \Omega_{\text {sym }}=0$ holds for the patterns built of the ellipses only. If we consider also the "interstitial" shapes emerging in the close-packed arranges (which are curvilinear quadrangles in Figure $\mathbf{5 c}$ and curvilinear triangles in Figure 5d) the informational measures of symmetry will be changed.

\subsection{Informational measures of symmetry of the mixed patterns built of $2 \mathrm{D}$ and $1 \mathrm{D}$ shapes}

The suggested information measures of symmetry are easily generalized for the mixed patterns containing 1D and 2D shapes such as the pattern shown in Figure 6, including $p$ irregular non-symmetric curves and $p$ equilateral triangles.

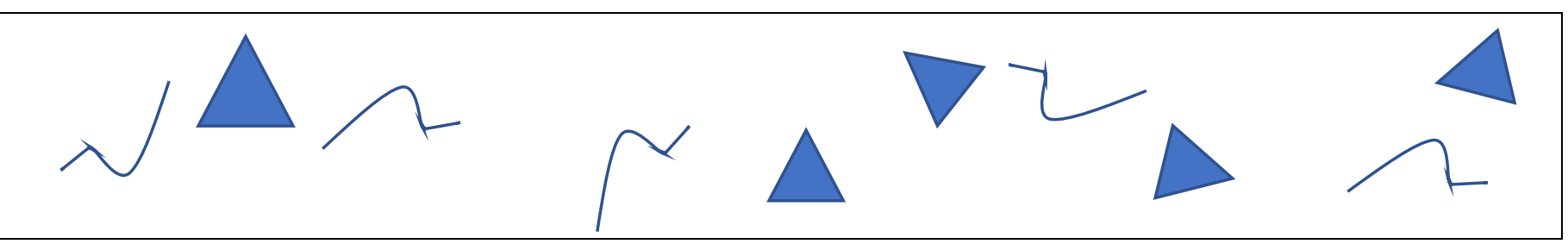

Figure 6. Mixed pattern built of $p$ irregular non-symmetric curves and $p$ equilateral triangles. 
Let us quantify the symmetry of this pattern. The symmetry group of the equilateral triangle is the dihedral symmetry group $\boldsymbol{D}_{\mathbf{3}}$ containing $3 p$ symmetry axes and $3 p$ rotations (including the $\mathbf{2} \boldsymbol{\pi}$ rotation, denoted $\boldsymbol{G}_{\mathbf{1}}$ ). One more $\boldsymbol{G}_{\mathbf{1}}$ operation comes, from the irregular curves; thus, we have in total $7 p$ symmetry operations in our pattern. Thus, IMS is easily calculated, according to $\boldsymbol{H}_{\text {sym }}=-\left(\frac{2}{7} \ln \frac{2}{7}+\frac{5}{7} \ln \frac{1}{7}\right)=\mathbf{2 . 2 3}$. Now we calculate VSMS of the same pattern; the probability to find within the pattern the shape possessing in total 6 elements of symmetry (triangles) equals $\frac{1}{2^{\prime}}$, the probability to find within the pattern the shape possessing the single element of symmetry (curves) also equals $\frac{1}{2^{\prime}}$ thus, we obtain $\Omega_{\text {sym }}=-2 \times \frac{1}{2} \ln \frac{1}{2}=0.69$.

\subsection{Informational measures of symmetry as dynamical variables}

Consider $N$ two-dimensional shapes (say ellipses and equilateral triangles shown in Figure 7) moving in a plane.

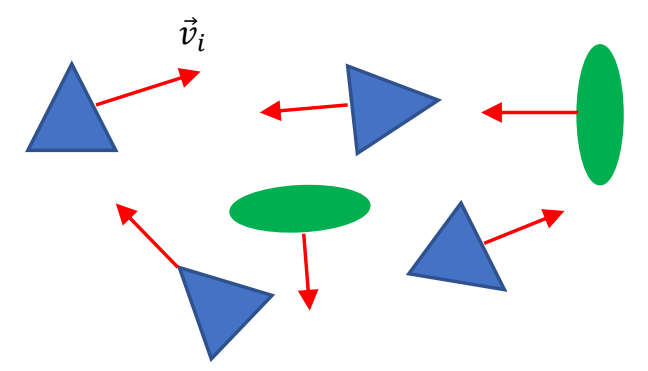

Figure 7. The planar movement of the ellipses and equilateral triangles is demonstrated. $\vec{v}_{i}$ is the velocity of the $i$-th shape.

The shapes (2D bodies) may collide elastically or stop their motion. If the symmetry of the bodies is conserved, we immediately conclude that the conservation laws $H_{\text {sym }}=$ const $; \Omega_{\text {sym }}=$ const take place. Thus, in this case conservation of the informational measures of symmetry takes place. Consider now the less trivial situation depicted in Figure $\mathbf{8}$ when $p$ rhombs shown on Figure 8a "dissociate" with time into $2 p$ equilateral triangles, shown in Figure $\mathbf{8 b}$.

\begin{tabular}{|l|l|}
\hline a) $H_{\text {sym }}=1.1 ; \Omega_{\text {sym }}=0$ & b) $H_{\text {sym }}\left(D_{3}\right)=1.792 ; \Omega_{\text {sym }}\left(D_{3}\right)=0$ \\
\hline
\end{tabular}

Figure 8. Time evolution of rhombs shown in Figure 8a, into equilateral triangles depicted in Figure $\mathbf{8 b}$ is depicted.

The initial Shannon measures of the system of rhombs shown in Figure 8a coincide with that of the ellipses already calculated in Section 2.2., namely $H_{\text {sym }}=1.1 ; \Omega_{\text {sym }}=0$. After dissociation into equilateral triangles we have $H_{\text {sym }}\left(D_{3}\right)=1.792 ; \Omega_{\text {sym }}\left(D_{3}\right)=0$ (see Section 2.2); this means that the change in the shape of objects constituting the pattern is quite expectedly accompanied with the jump in the informational measure of symmetry, accompanying the change in the symmetry group of the shapes. Hence, the introduced information measure of symmetry is well expected to be useful for characterization of phase transitions.

And last but not least: obviously the informational measures of symmetry could not be defined for the 0D objects (points). However, the set of points may be converted into the set of polygons with the use of the prescribed tessellation 
procedure (for example with the Voronoi tessellation [22-26]), and at the next stage the informational measures of symmetry of the tessellations may be calculated.

\section{Conclusions}

The paper addresses the fine structure of quantification of symmetry appearing in 2D patterns. Symmetry and ordering are usually quantified by the Voronoi entropy [22-26] and continuous measure of symmetry [17-21]. We introduced and calculated the informational measures of various patterns built of 1D (curves) and 2D (shapes) objects. One of the Shannon-like measures of symmetry labeled $H_{\text {sym }}(G)$ represents an averaged across the pattern uncertainty to find a symmetry operation, related to the symmetry group $G$, within the given pattern. The other Shannon-like measure of symmetry $\Omega_{\text {sym }}(G)$ is interpreted as an averaged across the pattern uncertainty to find the shape possessing in total $n$ elements of symmetry forming the group $G$, and it resembles the well-known Voronoi entropy [22-26]. We illustrated the introduced measures of symmetry with numerous patterns built of triangles, arcs, ellipses, rhombs and irregular curves, including the "mixed" patterns composed of 1D and 2D objects. The reported results strengthen the idea, that ordering and symmetry of the pattern could hardly by quantified with the single numerical value. We also calculated $H_{\text {sym }}$ and $\Omega_{\text {sym }}$ for the Serpinski fractal carpet built from equilateral triangles/squares. $H_{\text {sym }}$ and $\Omega_{\text {sym }}$ remain the same on all of the scaling levels of the Serpinski carpet. The introduced $H_{s y m}$ and $\Omega_{s y m}$ are the intensive parameters of the given pattern and they are insensitive to the number of 1D/2D shapes, their size and area of the pattern. Both of the measures are influenced by the groups of symmetry of the shapes, constituting the pattern. The time evolution of the Shannon measures of symmetry are considered. If the moving objects conserve their symmetry groups the informational measures of symmetry are time-independent. The time evolution of the Shannon measures of symmetry is illustrated with the example, in which rhombs dissociate into equilateral triangles, thus, changing the symmetry group of the pattern. It is well expected that the introduced Shannon measures of symmetry will be useful for the analysis of phase transitions. In our future investigations we plan to study: i) informational measures of symmetry (IMS and VSMS) of fractals; ii) change in the informational measures of symmetry inherent for phase transitions; iii) IMS and VSMS of time crystals [31], iv) 3D generalization of the introduced Shannon-shaped measures.

Author Contributions: Conceptualization, E.B.; M. F.; N. S.; methodology, E. B..; M. F.; N. S.; I. L.; S. S.; validation, M. F.; I, L.; formal analysis, E. B..; M. F.; N. S.; I. L.; S. S; investigation, E. B..; M. F.; N. S.; I. L.; S. S; resources, E.B.; S. S.; N. S.; data curation, E. B..; M. F.; N. S.; I. L.; S. S; writing-original draft preparation, E.B.; writing-review and editing, E. B..; M. F.; N. S.; I. L.; S. S supervision, N. S.; S.S, project administration, N. S., S.S. All authors have read and agreed to the published version of the manuscript to the work reported.

Funding: This research received no external funding.

Institutional Review Board Statement: Not applicable

Informed Consent Statement: Not applicable.

Data Availability Statement:

The data presented in this study are available on request from the corresponding author.

\section{Acknowledgments:}

The authors are thankful to Mrs. Yelena Bormashenko for her kind in preparing this manuscript.

The authors declare no conflict of interest.

\section{References}

1. Weyl, H. Symmetry; Princeton University Press: Princeton, NJ, USA, 1989.

2. Lederman, L., Hill C. T. Symmetry and the Beautiful Universe; Prometheus Books, Amherst NY, USA, 2005.

3. Van Fraassen, B.C. Laws and Symmetry; Oxford University Press: Oxford, UK, 1989.

4. Rosen, J. Symmetry in Science: An Introduction to the General Theory; Springer: New York, NY, USA, 1995.

5. Gross, D. J. The role of symmetry in fundamental physics, Proc. Natl. Acad. Sci. USA, 1996, 93, 14256-14259.

6. Haywood St. Symmetries and Conservation Laws in Particle Physics: An Introduction to Group Theory for Particle Physicists, Imperial College Press, London, UK, 2011.

7. Newnham, R. E. Properties of Materials: Anisotropy, Symmetry, Structure, Oxford University Press, Oxford UK, 2005.

8. Hall, B.C. Quantum Theory for Mathematicians. Graduate Texts in Mathematics; Springer: New York, NY, USA, 2013.

9. Tsukerblat, B. S. Group Theory in Chemistry and Spectroscopy, Dover Publications, Mineola, MY, USA, 2006. 
10. De Graef, M. Structure of Materials: An Introduction to Crystallography, Diffraction and Symmetry, 2nd Ed. Cambridge University Press, Cambridge, UK, 2012..

11. Chatterjee, S.K. Crystallography and the World of Symmetry; Springer: Berlin, Germany, 2008.

12. Selzer, M. Byzantine Aesthetics and the Concept of Symmetry, Independently published, ISBN-13: 979-8711275015, 2021.

13. Darvas, G. Symmetry: Cultural-historical and Ontological Aspects of Science-Arts Relations; Birkhauser: Basel, Switzerland, 2007.

14. Huang, Y., Xue, X., Spelke, E., Zheng, W., Peng K. The aesthetic preference for symmetry dissociates from early-emerging attention to symmetry. Sci. Rep. 2018, 8, 6263.

15. Hargittai, I.; Pickover, C.A. Spiral Symmetry; World Scientific: Singapore, 1992.

16. Fang, Y-N., ,Dong, G-H., Zhou, D-L., Sun, C. P. Quantification of Symmetry, Commun. Theor. Phys. 2016, 65, 423.

17. Zabrodsky, H.; Peleg, S.; Avnir, D. Continuous symmetry measures. J. Am. Chem. Soc. 1992, 114, 7843-7851.

18. Zabrodsky, H.; Peleg, S.; Avnir, D. Symmetry as a continuous feature. IEEE Trans. on Pattern Anal. Mach. Intel. 1995, 17, 1154-1166.

19. Pinsky, M.; Dryzun, Ch.; Casanova, D.; Alemany, P.; Avnir, D. Analytical methods for calculating Continuous Symmetry Measures and the Chirality Measure, Comp. Chemistry 2008, 29, 2712-2721.

20. Sinai, H.E.; Avnir, D. Adsorption-induced Symmetry Distortions in W@Au12 Nanoclusters, Leading to Enhanced Hyperpolarizabilities, Israel J. Chem. 2016, 56, 1076-1081.

21. Bonjack, M.; Avnir, D. The near-symmetry of protein oligomers: NMR-derived structures. Sci. Rep. 2020, $10,8367$.

22. Voronoi, G. Nouvelles applications des paramètres continus à la théorie des formes quadratiques. Deuxième mémoire. Recherches sur les paralléloèdres primitifs. Reine Angew. Math. 1908, 134, 198-287.

23. Barthélemy, M. Spatial networks. Phys. Rep. 2011, 499, 1-101

24. Bormashenko, Ed.; Frenkel, M.; Vilk, A.; Legchenkova, I.; Fedorets, A.A.; Aktaev, N. E. Dombrovsky, L.A.; Nosonovsky, M. Characterization of self-assembled 2D patterns with Voronoi Entropy. Entropy 2018, 20, 956.

25. Frenkel, M.; Fedorets, A.A.; Dombrovsky, L.A.; Nosonovsky, M.; Legchenkova, I.; Bormashenko, Ed. Continuous Symmetry Measure vs Voronoi Entropy of Droplet Clusters. J. Phys. Chem. C 2021, 125, 2431-2436.

26. Bormashenko, Ed., Legchenkova, I., Frenkel, M., Shvalb, N., Shoval, Sh. Voronoi Entropy vs. Continuous Measure of Symmetry of the Penrose Tiling: Part I. Analysis of the Voronoi Diagrams, Symmetry 2021, 13(9), 1659.

27. Bormashenko, Ed., Legchenkova, I., Frenkel, M., Shvalb, N., Shoval, Sh. Informational Measure of Symmetry vs. Voronoi Entropy and Continuous Measure of Entropy of the Penrose Tiling. Part II of the "Voronoi Entropy vs. Continuous Measure of Symmetry of the Penrose Tiling, submitted to Symmetry, 2021.

28. Ben-Naim, A. Entropy, Shannon's Measure of Information and Boltzmann's H-Theorem, Entropy 2017, $19(2), 48$.

29. Mandelbrot, B. S. The Fractal Geometry of Nature, W. H. Freeman and Co. NY, USA, 1983.

30. Peitgen, J. S. Chaos and Fractals, $2^{\text {nd }}$ ed., Springer, Berlin, Ge., 2004.

31. Wilczek, F. Quantum Time Crystals. Phys. Rev. Lett. 2012, 109 (16), 160401. 